\title{
Design Considerations for High Speed Quantitative Mass Spectrometry with MALDI Ionization
}

\author{
Jay J. Corr, Peter Kovarik, Bradley B. Schneider, Jan Hendrikse, \\ Alexander Loboda, and Thomas R. Covey \\ MDS SCIEX, Concord, Ontario, Canada
}

\begin{abstract}
A MALDI ion source on a triple quadrupole mass spectrometer constructed for the purpose of obtaining high speed quantitative measurements on drugs and other low molecular weight compounds is described. Particular attention is given to the ion generation and transport phenomena that affect analysis speed, throughput, and practical instrument robustness. In this regard parameters that affect desorption speed, beam spreading, ion flight times, sensitivity, signal-to-noise, ion fragmentation, sample carry-over, and instrument contamination are examined and experimental results are provided. MALDI and electrospray sensitivity is compared, to provide a practical frame of reference. (J Am Soc Mass Spectrom 2006, 17, 1129-1141) (c) 2006 American Society for Mass Spectrometry
\end{abstract}

$\mathrm{T}$ The development of orthogonal MALDI (OMALDI), which separates mass analysis from the laser timing, opened new opportunities for coupling MALDI to a variety of different mass analyzers [1]. One interesting configuration is MALDI on a triple quadrupole $(\mathrm{QqQ})$ designed for the express purpose of acquiring rapid quantitative measurements on drug entities and other low molecular weight compounds dried and arrayed on microtiter plates. The concept is analogous to plate readers used in high throughput screening albeit these optical based systems derive their speed from parallel measurements. In the case of a mass spectrometer the analysis is inherently serial in nature but could be operated at rates that would approach the throughputs of the optical systems. Assessments of the general utility of MALDI TOF for small molecule quantitative and qualitative analyses have been reported [2] and reviewed [3]. Studies of the practicality of the triple quadrupole approach for various applications have begun to appear in the literature using prototype triple quadrupole instruments $[4,5]$, which have examined in detail the quantitative figures of merit of the technique in biological samples, emphasizing the importance of the use of internal standards. In a sister publication to this work [4], issues important to quantitation and general analytical utility such as chemical diversity, background produced by blank measurements, and $\mathrm{s} / \mathrm{n}$ are covered in detail. The focus of this work are the characteristics of the instrumentation.

The reasons why a triple quadrupole is a logical

Published online June 5, 2006

Address reprint requests to Dr. T. R. Covey, MDS SCIEX, 71 Four Valley Drive, Concord, ON L4K 4V8, Canada. E-mail: Tom.Covey@sciex.com choice for a MALDI based high speed quantitation tool will be elaborated on in this paper but can be summarized as follows. MALDI on a triple quadruple decouples the synchronization of the desorption event with the mass analysis, similar to orthogonal MALDI TOF, but quite distinct from traditional low-pressure MALDI TOF. The mass spectrometer performance is thus independent from the sample morphology. This allows target preparation to be a noncritical issue so inhomogeneities in the sample, as would be expected in any high throughput methodology, will have no effect on the mass spectrometer mass resolution or accuracy.

High-pressure operation of the source and the preanalyzer RF optics is readily accommodated in a QqQ. Collisional cooling of the ion beam is achieved at higher pressures, which reduces ion fragmentation and allows a broader range of laser energy to be utilized. With low-pressure operation on TOF instruments the laser energy must be kept low, near the ionization threshold, to avoid fragmentation and to circumvent effects on mass resolution and accuracy.

With MALDI ionization the chemical noise is such that isobaric interferences exist in high abundance at virtually every mass, particularly in the low $\mathrm{m} / \mathrm{z}$ range. MS/MS is necessary to obtain the required signal-tonoise for quantitative measurements.

Transmission in a QqQ is high, roughly 35\% per mass analyzer. Duty cycle in the multiple reaction monitoring (MRM) mode approaches $100 \%$, depending on the number of ions monitored simultaneously, and measurement times less than $10 \mathrm{~ms}$ can be used. A tandem quadrupole TOF has the advantage of increased specificity from better product ion resolution and the collection simultaneously of all product ions 
simplifying method development, however, transmission is somewhat less. Duty cycle can be as low as $10 \%$ if a broad mass range is being captured due to variable flight times of ions of different mass in the orthogonal pulsing region, although improvements are possible if the mass range is limited [6]. Tandem in time instruments that require greater than $100 \mathrm{~ms}$ for MS/MS analysis, such as ion traps, will not be fast enough to profile the fast signal transients from a high repetition rate laser ion source. Trapping and accumulating the signal transients before injection into the analyzer trap is possible but will add time overhead to the analysis.

The dynamic range of a triple quadrupole is not encumbered to the same extent as many other types of mass analyzers. The limitations on quantitative dynamic range as a result of space charge effects in trapping analyzers are nonexistent in a QqQ, and would likely be severe from a source that produces the rapid, high intensity pulses expected from a laser operating in the $\mathrm{kHz}$ pulse rate range. Given the extensive chemical background generated with MALDI, a single laser pulse can exceed the space charge limits of a trap unless some ion pre-filtering is done. Although space charge can be ameliorated through the use of gain control techniques, these will reduce the duty cycle and reduce the ability to profile very fast signal transients.

The following report is a study of the relevant ion generation and transport parameters involved in the design of a MALDI ion source for a QqQ with particular attention to analysis speed, maximum pseudo molecular ion generation, freedom from sample carry-over effects, and practical instrument robustness. In this regard parameters that affect desorption speed, beam spreading, ion flight times, sensitivity, signal-to-noise $(\mathrm{s} / \mathrm{n})$, ion fragmentation, and sample ablation effects are examined and experimental results are provided. MALDI and electrospray sensitivity is compared, primarily because electrospray based methods are well understood and serve as a practical frame of reference. Quantitative figures of merit such as reproducibility, linearity, and dynamic range are not covered as they have been addressed in a previous publication [4].

\section{Experimental}

All experiments were performed on a modified API 4000 triple quadrupole or a modified 4000 Q TRAP [7]. The instruments were operated in the MRM mode with unit resolution in both quadrupoles. The accelerating axial field applied to the collision cell in the standard instrument (LINAC) $[8,9]$ is an important aspect of the analyzer for this application where ion flight times and beam spreading will present the fundamental limits to sample throughput. The channeltron detector uses pulse counting circuitry so all ion current measurements are expressed in counts per second (cps) corresponding to ion events per second.

The primary modifications to the instrument described herein were implemented in the ion source and



Figure 1. (a) Experimental MALDI ion source design. (b) Modification to add experimental flexibility to the laser angle of incidence and target spacing distance from the aperture. All aspects of (b) are the same as (a) except the position of the laser and the use of mirrors to redirect the beam.

transfer optics regions (Figure 1) similar to those described in [10]. Q0 is the standard API 4000 quadrupole $\mathrm{Rf}$ only ion guide spaced $4 \mathrm{~mm}$ from the source aperture. The standard $800 \mathrm{~L} / \mathrm{s}$ turbopump, used for electrospray sources, was mounted on this region but provided excessive pumping for the requirements of this source so auxiliary gasses were required. The operating pressure of the ion source and Q0 regions were adjustable with nitrogen gas through needle valves. Source pressures from 0.1 to 10 torr could be accessed while maintaining the Q0 pressure in a range of 3-10 mTorr pressure to maintain ion beam focusing [11] and maximize transfer through a $1.4 \mathrm{~mm}$ aperture into the $10^{-5}$ torr analyzer region.

A $4 \mathrm{~mm}$ diameter aperture separated the Q0 rods from the source. The target was spaced 1.5 to $16 \mathrm{~mm}$ from the aperture; this distance was varied during the course of the experiments. The source pressure range was $0.1-5$ torr, adjustable with nitrogen gas through a needle valve. The aperture had a sliding mechanism that allowed it to serve as a vacuum gate. The ion source region could be opened to atmosphere to afford target replacement and presented the target to the outside world in a horizontal plane amenable to automated plate transfer equipment. The aperture, motion stages, and other optics in this region were thus exposed if cleaning or routine replacement was required or an electrospray interface was to be mounted without breaking vacuum. Pump down to operational pressure required $\sim 20 \mathrm{~s}$ using a $16 \mathrm{~m}^{3} / \mathrm{h}$ roughing pump. A full cycle of venting, plate loading, and pump down was 
less than $1 \mathrm{~min}$. The source opening motors as well as the laser attenuator was controlled by a Parker Hannifin ViX-IM controller (Holis, New Hampshire), the same controllers used to drive the $\mathrm{XY}$ stages (discussed below).

A JDS Uniphase $355 \mathrm{~nm}$ solid-state laser (Nanolase SC1, Grenoble, France) adjustable to $1400 \mathrm{~Hz}$ pulse rate and available power of $15 \mu \mathrm{J}$ per pulse was used in this work. Figure 1a shows the laser mounted on the vacuum chamber in the Q0 region, directed between the Q0 rods and offering a direct line of sight to the target at a low angle of incidence, relative to the target normal. Figure $1 \mathrm{~b}$ shows the laser mounted on the source region to allow more flexibility for laser incident angle adjustment and target spacing from the aperture. A CCD camera with a $4 \mathrm{~mm}$ field of view and a LED light mounted on both of these regions of the instrument were used to illuminate and visualize the target. Optical fibers were coupled to direct the beam in most experiments, however the design of Figure 1a allowed for direct illumination of the target and the avoidance of energy losses due to fiber aging and to eliminate coupling problems. The typical spot diameter with a $30^{\circ}$ angle and focusing lenses was $150 \mu \mathrm{m}$ but was modified during the course of the experiments with fibers.

The XY stage was based on Parker Hannifin (Daedal division) MX80 series of miniature stages equipped with NEMA micro stepping electric motors and a ball screw lead drive capable of addressing all coordinates of an SBS microtiter format target, all materials selected for vacuum compatibility. The $X$ and $Y$ motors deliver a maximum of 98 and $120 \mathrm{~N}-\mathrm{m}$ torque, respectively. The system was limited by resonance and torque roll at high speeds to a maximum speed of $30 \mathrm{~mm} / \mathrm{s}$ and acceleration of $3 \mathrm{~m} / \mathrm{s}^{2}$, but delivered actual timed jumps of 90 ms over $9 \mathrm{~mm}$ distances ( 96 well format) and $60 \mathrm{~ms}$ over $4.5 \mathrm{~mm}$ distances (384 well format) with any extreme point on the target accessible in under $3.0 \mathrm{~s}$. Faster stages are available based on piezo or magnetic levitation principles but these stepper motors were deemed adequate for the purposes of this study as throughput was limited by beam spreading in the analyzer rather than stage motion. The micro stepping motor resolution was 50,000 steps per revolution with a positional accuracy of $0.5 \mu \mathrm{m}$ per $10 \mathrm{~mm}$ of travel. The stage was controlled through two "daisy chained" ViX-IM controllers linked to a host computer by an RS 232 connection. Since samples were deposited in a Cartesian grid format, independent two axis control was deemed adequate for this quantitation application. Other applications such as liquid chromatography trace reading and tissue imaging may require axis tracking control to maintain constant velocity around curved paths. The mechanical tolerances of the source, stage, and laser optics were held so as to be able to locate any coordinate on a target to within $0.1 \mathrm{~mm}$ with both accuracy and precision. Since typical spot sizes ranged from 1 to 3 $\mathrm{mm}$ in diameter, samples could be reproducibly located and interrogated by virtue of their mechanical coordi- nates, negating the absolute necessity for machine vision systems to locate samples which would slow down read rates.

The primary method of acquisition was MRM, where a single region of a sample spot the size of the laser illumination area was ablated until the sample was depleted from the target. This mode of sampling is referred to as complete "burning through". Partial burn through is also done by controlling the laser ionization time from $2 \mathrm{~ms}$ to $1 \mathrm{~s}$. After a burn the stage was moved to the next sample, acquiring the next quantitative measurement in the same fashion. An alternative method was to run the stage at a constant velocity with the laser on at all times. This rastering method has particular application for reading chromatograms.

Spotting samples on the target was done by one of several methods. The dried droplet technique was used with either manual deposition or with the use of a precision electrostatic deposition device [12]. Eksigent Express 100 pumps (Dublin, CA) delivered the mobile phase for the chromatographic separations synchronized with the spotter by a computer data system [13]. This instrument was designed to record chromatographic traces on MALDI targets (TempoLC/MALDI, Applied Biosystems/MDS SCIEX, Foster City, CA). For some of the LC/MALDI experiments a gas nebulizer substituted for the electrostatic deposition device (Meinhard HEN-170-AA, Santa Anna, CA) with an unheated gas flow of $1 \mathrm{~L} / \mathrm{min}$. Publications involving the use of heated gas flow nebulizers have been reported [14].

Various MALDI matrices were tested, but $\alpha$-cyano4-hydroxycinnamic acid (CHCA) offered the best combination of wide applicability across the small molecule chemical space, homogenous crystallization and commercial availability $[4,5]$. The current experiments were performed with CHCA solutions $(6.2 \mathrm{mg} / \mathrm{ml}, 36 \%$ methanol, 56\% ACN, pH 2.5) available from Agilent Technologies (Palo Alto, CA) part number G2037A. Standard solutions were typically made in 50/50 acetonitrile/water and mixed 1:1 with the matrix solution deposited in 0.25 to $3.0 \mu \mathrm{L}$ volumes. Before spotting, the stainless steel targets were sonicated in methanol, then water, and polished. The matrix was found to etch the target surface over time causing deterioration in the ability to accurately deposit a spot with plate re-use. This effect was minimized by applying a uniform hydrophobic coating over the plate surface before each re-use, in this case POL metal polishing paste (SPI supplies, PA). An alternative method is the plasma etching/Teflon coating technique of Ericson et al. [12]. All other chemicals were obtained from Sigma Chemical Co. (St. Louis, MO).

\section{Results and Discussion}

For high speed quantitative analysis the goal is to generate maximum pseudo molecular ion signal intensity as fast as possible. An investigation was undertaken 
to study the characteristics of an ion source, laser, and transfer ion optics on a triple quadrupole to achieve this goal for low molecular weight compounds. Other characteristics, relevant to the intended application, were also studied such as those that could lead to sample-tosample carry over effects and instrument contamination over time.

\section{Laser Fluence and Collisional Cooling of Ions}

For CHCA matrix the threshold energy for ion production was reported to be $\sim 1.5 \mu \mathrm{J} /$ pulse for proteins [15]. It has long been known in the MALDI TOF field that it is desirable to increase the energy per pulse to slightly above the ionization threshold to improve sensitivity. Increasing the energy will also lead to pseudo molecular ion fragmentation. Increasingly, energetic ions were formed that either fragment in the ion source or fragment by metastable decay in the flight tube and reflectron. The useful energy range is narrow, between 1.5 and 2 times the ionization threshold, and finding the balance between sensitivity and fragmentation in this narrow energy range has always been an important aspect of MALDI TOF instrument tuning.

Collisional cooling has been shown to suppress fragmentation of ions in MALDI; the internal energy of the ion is relaxed in gas collisions. The 3-100 mTorr pressures in an RF ion guide transferring ions from a MALDI source to the analyzer cool ions to a significant degree post ionization [1]. Cooling ions with a high gas density in the immediate region of the ablation plume provides an additional cooling effect decreasing fragmentation at higher energy thereby improving sensitivity [15-17]. Detailed studies have arrived at an optimal source pressure of $\sim 1$ torr that provides a good compromise between maximum cooling rate and minimum matrix adduct formation [18]. It also allows for the use of matrices that have high phase transfer temperatures. These so called "hot matrices", such as CHCA, have been shown to provide a more universal response for small molecules $[4,5]$.

For small molecules we observe a relatively low ionization threshold, typically 0.7 to $1 \mu \mathrm{J} /$ pulse. Peak signal intensities were obtained without fragmentation in an energy range from 1.5 to 5 times the threshold energy, roughly from $2-5 \mu \mathrm{J} /$ pulse, when operating the source and Q0 pressures at 1 torr and 7 mTorr, respectively. This operating range was observed for both low and high repetition rates. When quantitation and throughput are the goal of analysis, it is beneficial to have a broad energy range in which to operate. A laser that provides energies per pulse beyond this range was not required. However, operating at high laser repetition rates within this energy range will have a beneficial effect on both the total ion production and the rate of its formation as will be discussed further in a subsequent section of this paper.

To demonstrate the significance of the source cooling effect with small molecules, a compound known to
Table 1. Effect of source gas pressure on fragmentation of Minoxidil measuring the ratio of the product to precursor ion intensity. The laser pulse frequency $=1400 \mathrm{~Hz}$ and energy per pulse $=4.5 \mu \mathrm{j} /$ pulse

\begin{tabular}{cc}
$\begin{array}{c}\text { Source pressure } \\
\text { (Torr) }\end{array}$ & $\begin{array}{c}\text { Ratio of the fragment to Pseudo } \\
\text { molecular ion intensity, m/z } \\
193 / 210\end{array}$ \\
\hline \hline 0.3 & 0.9 \\
0.4 & 0.7 \\
0.6 & 0.2 \\
1.0 & 0.1 \\
1.2 & 0.1 \\
1.4 & 0.1 \\
\hline
\end{tabular}

have a low fragmentation threshold was chosen. The Minoxidil parent ion $\left(\mathrm{M}+\mathrm{H}^{+}=210\right)$ readily undergoes a facile cleavage $(\mathrm{m} / \mathrm{z}=193)$ at low collision energies $(<10 \mathrm{eV})$. The ratio of these two ions was monitored as the source pressure was varied by bleeding nitrogen into the source. An additional gas bleed into the Q0 region made it possible to maintain fixed pressure conditions downstream of the source region for these experiments. The Q0 pressure optimized over the range of 3 to $8 \mathrm{mTorr}$. The data in Table 1 shows a 9-fold suppression of fragmentation as the source pressure varied from 0.3 to 1.4 torr. The ion current from the pseudo molecular ion increased proportionally to the decrease in fragmentation. The laser energy was maintained at $4.5 \mu \mathrm{J} /$ pulse but produced similar trends within a range of 2 to $7 \mu \mathrm{J} /$ pulse.

Having established the initial critical pressure and energy parameters, all further experiments were performed at source pressures of 1 torr and Q0 pressures of 7 mTorr. An aperture diameter of $4 \mathrm{~mm}$ separating these regions was chosen to restrict the gas flow without decreasing ion flux from the source. Laser energies were kept to within 2-7 $\mu \mathrm{J}$ per pulse. The effects of laser repetition rate and other source parameters were then examined.

\section{Effect of Laser Repetition Rate on Speed, $s / n$, and Total Ion Yield}

The laser repetition rate has a significant influence on the speed at which quantifiable signals can be generated. The majority of the work to date has focused on using high repetition rate capability to match the pulse rate with the flight time of a TOF analyzer to increase the number of high quality spectra per second $[15,16]$ or to produce a continuous beam of ions for use in an orthogonal TOF instrument configuration [19]. The high repetition rate laser was used for a different purpose in this work. The concept was to produce a high intensity packet of ions as quickly as possible to use as a measure of the quantity of material on the target. As mentioned earlier, the laser illuminates an area of the sample spot and remains in that position until the sample is burned through to the target and the signal decays. An example 



Figure 2. Signal transients from firing a laser at four different locations of a sample spot then repeating this process on a separate sample spot. (a) Laser at $1400 \mathrm{~Hz}$ (4 $\mu \mathrm{J} /$ pulse), MRM dwell time $=10 \mathrm{~ms}, 4$ replicates on two different spots. (b) Laser at $10 \mathrm{~Hz}, \mathrm{MRM}$ dwell time $=100 \mathrm{~ms}$. Four replicates on one spot. The inserted image is that of the first sample spot with the four replicate burns taken at $1400 \mathrm{~Hz}$. In both cases the laser remained firing until the sample was depleted in the illuminated area. The analyte was a $10 \mathrm{pg} / \mathrm{ul}$ solution, $1 \mu \mathrm{l}$ sample spot of Prazosin monitoring $\mathrm{m} / \mathrm{z}$ $384 \rightarrow 247$.

of data acquired in this fashion demonstrating the effect of laser repetition rate is shown in Figure 2a and $b$. In Figure 2a, the laser was operated at $1400 \mathrm{~Hz}$ and illuminated four separate areas of a single sample spot before repeating this process on the next sample spot. These four separate illuminations could be considered replicate measurements of the same sample. The signal transients or "peaks" achieve a full width at half maximum (FWHM) and base width of $\sim 65$ and $600 \mathrm{~ms}$, respectively. Figure $2 b$ was taken at the same laser fluence and $10 \mathrm{~Hz}$. The maximum rate of ion liberation was 70-fold lower than at $1400 \mathrm{~Hz}$ with base width 60 -fold greater. The average peak areas for the high repetition rate data were $50 \%$ greater than the low repetition rate data, and both the $\mathrm{S} / \mathrm{N}$ and speed at which a measurement could be taken were improved. More well defined signal transients were created, whose areas could be easily integrated. This general trend was observed with several hundred compounds, all drug-like entities.

Figure 3 shows a plot of the peak FWHM versus laser repetition rate for a different compound, Haloperidol. The FWHM decreased from $\sim 17 \mathrm{~s}$ at a laser pulse rate of $10 \mathrm{~Hz}$ to $\sim 0.1 \mathrm{~s}$ at a laser pulse rate of $1400 \mathrm{~Hz}$, nearly 200-fold. As with the data for Prazosin in Figure 2, the decrease in peak width was reflected as an increase in peak height as shown in Figure 4. An overall increase in peak area of $50 \%$ was observed indicating slightly

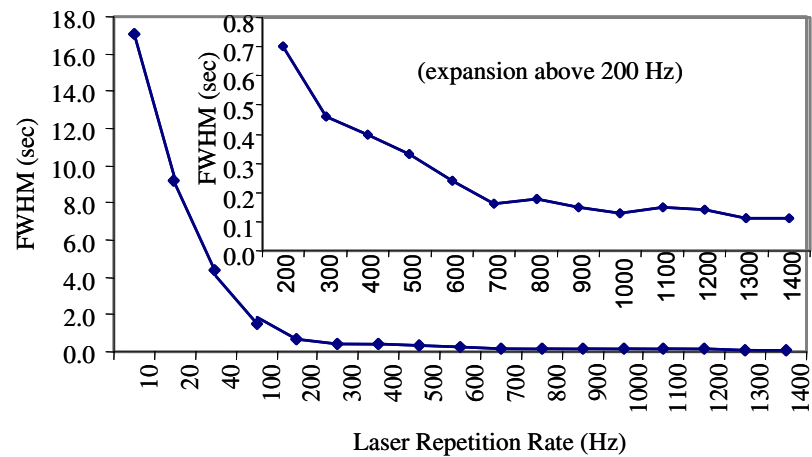

Figure 3. Plot of the FWHM of the signal transients at various laser repetition rates with constant energy of $6 \mu \mathrm{J}$ per pulse. The analyte was a $25 \mathrm{pg} / \mu \mathrm{L}, 1 \mu \mathrm{L}$ spot of Haloperidol $(\mathrm{m} / \mathrm{z} 376->165)$ analyzed according to the method of acquisition described in the text. 


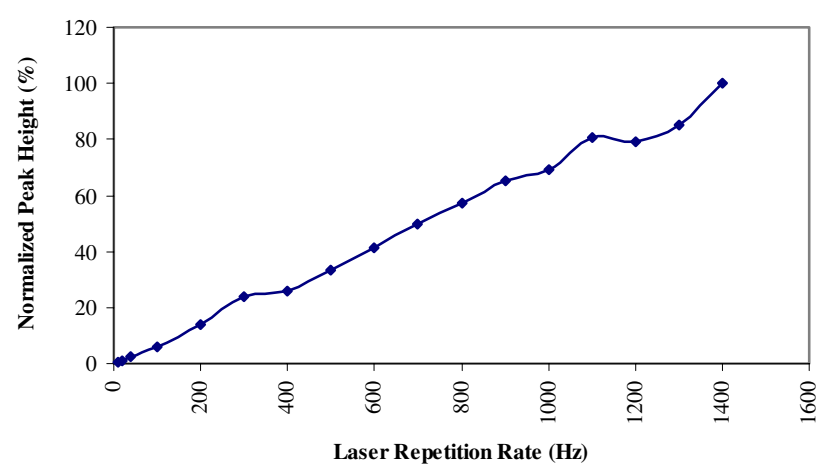

Figure 4. Plot of the peak height in counts per second of the signal transients from firing the laser at various repetition rates with a constant energy of $6 \mu \mathrm{J}$ per pulse The sample was a $1 \mu \mathrm{L}$ spot of $25 \mathrm{pg} / \mu \mathrm{L}$ Prazosin monitoring $\mathrm{m} / \mathrm{z} 384->247$.

higher ionization efficiency at higher repetition rates. Comparing Figures 2 and 3, there were significant differences in peak widths for the different compounds. There is a compound dependency with respect to the time for the sample to be completely ablated. However what appears to be consistent from compound to compound is the number of laser shots to achieve maximum peak intensity. A means to compensate for this compound dependency in signal decay rates is discussed in the next section.

\section{Effect of Laser Gate Time on Speed of Analysis}

Liberation of ions from the target surface during illumination does not occur at a constant rate during the ablation process. Liberation rates are initially high and then slow down resulting in tailing peaks. There is a significant degree of compound dependency with regards to this behavior, the chemistry of some compounds inducing longer decay times. What is universally observed is all compounds are liberated at high rates within the first $10 \mathrm{~ms}$ but some resist total ablation to zero signal and produce long lingering tails, a potential problem for routine high throughput analysis. A study was done to attempt to eliminate this problem by controlling the laser illumination time, acquiring only the data from the initial burst of ions and turning the laser off during the subsequent signal decay.

An example of such an experiment is shown in Figure 5 for Haloperidol. This compound showed signal transients greater than $1 \mathrm{~s}$ at baseline when subjected to irradiation until sample depletion. Shortening of the irradiation time to $10 \mathrm{~ms}$ had no effect on the peak height but predictably truncated the signal transient (Figure 5). Repeated runs at a range of concentrations indicated the peak area calculations were as repeatable as if the samples were allowed to ablate to total depletion, providing a means to decrease the acquisition time and hence throughput.

Compound dependency with regard to the rate of the signal decay during the ablation of a region of a spot could vary by an order of magnitude. Rather than

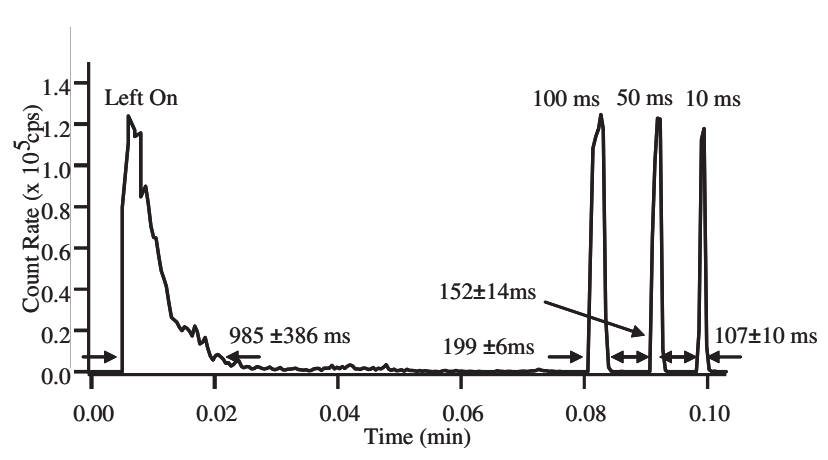

Figure 5. The effect of reducing the laser illumination time on the signal transients for Haloperidol. Laser pulse frequency was 1 $\mathrm{kHz}$.

ablate the sample completely to the target, this method of partial ablation was used to make the acquisition time equivalent for all compounds, this being possible because peak signal intensity was reached of all compounds within $10 \mathrm{~ms}$. Regardless of variations in chemistry a constant and reproducible peak width of $\sim 100$ ms could be achieved with illumination times of $10 \mathrm{~ms}$ (10 laser shots at $1 \mathrm{kHz}$ ) as shown in Table 2 .

Gradually reducing the irradiation time and measuring the signal also allows an estimate of the contribution to the peak broadening from the ion optics which establishes the fundamental limitation to the sample speed. It also provides some indication of the number of laser shots required to achieve peak signal intensity. Referring to Figure 5, maximum peak intensity remained constant down to illumination times of $10 \mathrm{~ms}$ (10 laser shots) and there was a linear relationship between observed peak width and illumination time. Reducing the illumination time to values less than 10 $\mathrm{ms}$ began to reduce the maximum peak intensity but the peak width remained constant at $100 \mathrm{~ms}$. This provides a measure of the peak spreading during transit from the target to the detector of $\sim 100 \mathrm{~ms}$. Similar measurements for the four other small molecules also showed limits of $\sim 95-100 \mathrm{~ms}$ per sample, all reaching maximum peak intensity in $10 \mathrm{~ms}$. This establishes the theoretical throughput of this instrument at 10 samples per second as limited by the ion beam spreading. The majority of the ion flight time and beam spreading occurs in the high collision frequency regions such as Q0. Shortening the Q0 region, applying an axial field, or establishing a field gradient using stacked

Table 2. MRM base peak widths (ms) with various irradiation times for various compounds

\begin{tabular}{lllll}
\hline \multicolumn{1}{c}{ Sample } & \multicolumn{1}{c}{ Left on } & \multicolumn{1}{c}{$100 \mathrm{~ms}$} & \multicolumn{1}{c}{$50 \mathrm{~ms}$} & \multicolumn{1}{c}{$10 \mathrm{~ms}$} \\
\hline \hline Clonidine & $642 \pm 196$ & $194 \pm 10$ & $145 \pm 18$ & $111 \pm 8$ \\
Haloperidol & $985 \pm 386$ & $199 \pm 6$ & $152 \pm 14$ & $107 \pm 10$ \\
Ketoconazole & $643 \pm 51$ & $187 \pm 9$ & $151 \pm 10$ & $114 \pm 8$ \\
Prazosin & $734 \pm 266$ & $201 \pm 6$ & $147 \pm 9$ & $111 \pm 10$ \\
Quinidine & $954 \pm 150$ & $201 \pm 5$ & $141 \pm 5$ & $109 \pm 6$ \\
\hline
\end{tabular}


lenses are all possible means to reduce the spreading and improve the theoretical throughput.

\section{Effect of Laser Angle of Incidence on Sample Carry-Over}

The laser incident angle, within certain limits, is not expected to affect signal intensities [20], and experiments with this source indicate that this is the case. A laser angle, relative to the target normal, of less than $30^{\circ}$ results in a nearly circular burn spot while angles of $60^{\circ}$ result in an elliptical laser burn with a major to minor axis ratio of $\sim 2.6$ to 1 , the dimension of the minor axis is given by the size of the laser beam diameter and the magnification and aberration provided by the laser focusing system. A greater angle of incidence causes the burn mark to be stretched further in one dimension requiring the use of higher laser power to provide sufficient fluence over the irradiated spot to achieve the same sensitivity as a circular burn with the same area. Excessive angles should therefore be avoided to conserve laser power and assure constant fluence.

There have been reports that the laser incident angle will affect the trajectory of the ablation plume such that the plume follows a path toward the incident laser light [21]. The greater the laser angle from normal to the target the greater the deviation of the ablation plume from the same normal. We have observed that the incident laser angle does affect the ablation plume trajectory and the consequences can be considerable for quantitative applications when considering the possibility of cross contamination of sample spots.

In some situations, airborne particles of sample and matrix can be visually observed to land on adjacent spots under the magnification of the CCD camera. The degree to which this is observed increases as the laser incident angle increases. Experiments were performed to measure the degree of cross contamination at angles of $62^{\circ}$ and $25^{\circ}$ from normal to the target at a laser energy of $6 \mu \mathrm{J} /$ pulse and $1 \mathrm{kHz}$ pulse frequency. At the larger angle contamination of spots within $5 \mathrm{~mm}$ to those representative of high sample quantities (tens of nanograms) exhibited signal levels 3- to 6-fold above the blank (matrix and no analyte). Higher sample densities made the carry-over worse as did higher laser fluence. At an angle of $25^{\circ}$ the plume lifted off close to normal to the target surface and no measurable cross contamination was observed. The compound used for this study was Prazosin in the standard CHCA matrix. It can be expected that different matrices will exhibit different behavior in this regard.

\section{Fiber Diameter and Illumination Area Effects}

The larger the illumination area the greater the number of ions will be extracted per pulse provided the energy density is not reduced and the illumination area does not exceed the ability of the ion optics to accept the ablation plume. Experiments increasing the illumination area showed a decrease in the rate of improvement in ion transfer into the ion optics as one exceeds $200 \mu \mathrm{m}$ and approaches $400 \mu \mathrm{m}$ laser spots sizes beyond which the fluence drops below the optimum required for maximum sensitivity. The energy available from the laser is also a limiting factor. Other reasons to limit the illumination area to $200 \mu \mathrm{m}$ or less are to avoid reduction of chromatographic resolution when reading chromatographic traces deposited on a target and to avoid potential complications from analyte/matrix clustering that maybe more likely to occur with larger illuminated spot sizes [18].

\section{Ion Transfer from Target to Analyzer Using Gas Flow and Electric Field Focusing}

In the system described here, where there are regions of differential pressure, two physical forces are available to direct the ions from the target into the $\mathrm{Q} 0$ ion guide, gas drag and electric fields. The two have distinct properties; most notably gas drag will move both charged and neutral particles whereas electric fields will move charged particles alone. Their combined effects can be used to advantage to achieve high transfer efficiency for ions and maintain some degree of discrimination between ions and neutral particles.

Optics and analyzer contamination is of particular concern for an instrument whose purpose is high sample loads. Some opportunity to discriminate ions from neutral particles appeared conceivable in light of imaging studies of the ablation plume [22]. Although these published images were done at lower pressures and larger illumination areas (similar energy densities) they indicate that the trajectories of matrix and analyte differ and give some first approximations of plume dimensions. With these first estimates in mind, experiments were designed to see if a preferential transfer of ions over matrix contaminants could be achieved, taking advantage of the viscous gas drag forces, derived from the pressure differential of the source and Q0 optics region, and focusing electric fields.

The imaging results gave a strong indication that some analyte ions occupy the central axis of the plume while a large portion of the matrix rapidly diverges in the radial direction. Gas drag forces immediately in front of the aperture are strong and will efficiently draw both ions and neutrals through the aperture. The pressure differential across the aperture isolating these two regions is sufficiently high that the mean free path between collisions is much shorter than the $4 \mathrm{~mm}$ orifice diameter so losses due to collisions with the walls are insignificant. The subsequent gas expansion injects the ions and neutrals several $\mathrm{cm}$ into the Q0 rods at roughly sonic velocities. Free jet gas dynamic calculations [23] and measurements of MALDI plume speeds [22] approximate the transit time from the target to the Mach disc $\sim 3 \mathrm{~cm}$ into the rods to be about $0.1 \mathrm{~ms}$. 
Positioning the target close to the aperture assures efficient transfer of ions however there is no discrimination between ions and neutrals. Spacing the target outside the immediate influence of the gas flows and using electric fields to direct the ions toward the gas flow streams presents an opportunity to discriminate between ions and neutrals. A significant proportion of the neutral components of the rapidly diverging matrix plume can be pumped away by the vacuum system if positioned outside the influence of the gas flow. The importance of these considerations is to find a reasonable balance between high transmission efficiency operating entirely within the gas flow dominated region and reduced transmission efficiency with electric field focusing but maintaining some degree of discrimination between ions and neutral particles. Experiments were conducted to see if this discrimination between ions and neutrals could be achieved.

Three experiments were conducted situating the target at varying distances from the aperture to move the ablation plume in and out of the region where vacuum drag exerts an influence. Absolute signal intensities were used as a measure of efficiency of ion transfer. Measuring the extent of neutral particle discrimination was more difficult and involved ablating a large number of samples and tracking the deposition of neutral material on the aperture and Q0 rods by both visual inspection and as indicated by a loss of signal intensity due to charging of the ion optics over many ablation events. Defining "one sample" as the ablation of all material from one location on the target plate, samples were consecutively ablated until the absolute signal intensity fell to below $50 \%$ of starting conditions. The experiments were repeated on two different drug species using the standard CHCA matrix.

The first experiment used a $2 \mathrm{~mm}$ spacing, where the entire plume would be within the gas entrainment area and it showed the highest efficiency of transfer of all the experiments. This configuration was also the most susceptible to contamination, showing distinctly visible deposits on the orifice and Q0 rods, and heavy contamination on the Q0 exit aperture after 30,000 sample ablations at which point a 50\% signal reduction was observed. No voltage potentials directing the ions toward the aperture were required as would be expected in a totally gas flow dominated condition. The freedom from requiring a voltage could be considered a benefit of this approach but it comes with a cost.

The second experiment used $4 \mathrm{~mm}$ spacing and demonstrated absolute signal intensities $60 \%$ of that achieved in the first experiment. It was, however, less susceptible to contamination, showing a $50 \%$ reduction of the initial signal after an average of $\sim 40,000$ samples. A $10 \sim 20 \mathrm{~V}$ potential difference between the target and aperture was required to drive the ions toward the aperture, but did not show any mass dependency, allowing it to remain at a fixed potential. This result suggested that a portion of the ablation plume was outside the gas drag region, permitting a portion of the contaminants to be pumped away. As described in the section on carry-over, the laser incident angle affects the trajectory of the ablation plume. Positioning the plume outside the gas drag region is therefore a function of both the distance from the aperture and the laser incident angle.

The third experiment used $16 \mathrm{~mm}$ spacing. Optics contamination was significantly reduced relative to the others, requiring $>200,000$ samples to achieve a $50 \%$ signal reduction. The drift potential between the target and aperture required higher voltages than the second case as expected, a range of 50 to $150 \mathrm{~V}$. With the optimum voltage applied the signal intensities were similar to experiment 2 with no measurable losses due to the added flight length. At this distance the plume was well outside the gas drag region and approaching the conditions described by Gillig et al. [24], where radial diffusion of ions in a $12 \mathrm{~cm}$ mobility cell, at similar pressures to those used here, was observed to be minimal. This appeared to be the case with this experimental set-up as well and can be used to advantage as a means to discriminate between neutrals and ions. Ions travel through the drift region with some but relatively few losses due to spreading between the target and aperture and some of the neutrals were pumped away before reaching the strong vacuum draw region close to the aperture.

Discriminating between neutrals and ions is critical for an instrument geared toward very high sample speeds, numbers, and long-term instrument reliability. The ion mobility approach described above provides reasonable results. Other means were also investigated as described below.

\section{Ion Transfer from Target to Analyzer Using High-Pressure Ion Guides}

One means of achieving beam collimation and focusing over long distances at high pressures is to use an $\mathrm{Rf}$ ion guide, taking advantage of the collisional focusing principle [11] in a manner similar to the optics in the Q0 and collision cell regions, but at nearly $100 \times$ higher pressure.

Rf multipoles operated at high-pressure are efficient devices for radially containing ions. However, these devices present an axial field free region where ions, traveling through high-pressure regions, are slowed down to the point that they will come to a near stop unless aided in some manner. This presents no particular problem for continuous beam ion sources such as electrospray where ions are both launched at substantial velocities into the rods by gas free jet expansions and the continuous beam itself pushes the leading ions by the trailing ions using space charge forces. Transit times through the 8 mTorr Q0 region of this instrument have been measured to be about $10 \mathrm{~ms}$ from a pulsed electrospray source where the ion axial motion is derived mainly from the free jet expansion of gas into this 




Figure 6. Modification of the MALDI source in Figure 1 with an $\mathrm{RF}$ quadrupole ion guide in the high-pressure ion source region and gas directing sleeve.

device. The flight times through Rf rods at 1 torr is considerably longer, particularly when the ions are not launched into the rods by a gas expansion but instead come from a MALDI ablation event. Of equivalent concern is the spreading of the ion pulse in the axial direction within the device. It has been observed that a full burn through of the sample with the laser will produce a signal that will last a few seconds through such a device.

Both speed of analysis and signal to noise can be severely affected under such circumstances. The use of an axial field (LINAC) has been described to counter this effect in 1-10 mTorr collision cells similar length to that used here, demonstrating less than $1 \mathrm{~ms}$ transit time with an axial voltage drop of $\sim 2 \mathrm{~V}$ [25]. However, operation of an ion guide at 1 torr requires unrealistic voltages for a LINAC to overcome the velocity loss due to the large number of collisions. Estimates from the ion mobility literature can be used to approximate the voltages required. A 1-10 torr mobility analyzer was described by Gillig et al. [24], which required a field of $30 \mathrm{~V} / \mathrm{cm}$. A $3 \mathrm{~cm}$ LINAC would require the equivalent of $90 \mathrm{~V}$. Because the DC field penetration of the RF rods is roughly $1 \%$ of that achieved with the stacked rings of a mobility cell $\sim 9000 \mathrm{~V}$ would be required to move the ions with this approach. The application of $9000 \mathrm{~V}$ is unrealistic in the low torr pressure regime due to discharge. A highly segmented quadrupole device [26] could be a possibility but might present a higher degree of complexity.

Another means to drive the ions down the RF multipole is to create a net gas flow in the direction of the aperture (Figure 6). A gas restricting sleeve around the multipole directs the flow of background gas along the length of the rod array toward the aperture. A roughing pump port was attached directly to the back of the sleeve to generate a net gas flow of $\sim 33 \mathrm{~m} / \mathrm{s}$, dragging ions along its length in $\sim 3 \mathrm{~ms}$. Figure 7 shows MRM data collected for a sample of Haloperidol using a $10 \mathrm{~cm}$ RF multipole with and without gas directing sleeve. The data were acquired in the same fashion as described above, with a complete burn through. Flight times were improved but beam spreading occurred and produced peaks that were substantially wider than those shown above for the aperture configuration of Figure 1. However, the addition of the gas flow did show a beneficial effect and points a direction for future improvements such as shorter rods coupled with directed gas flows.

RF multipoles show some promise but a mobility approach is the most straightforward. A simple mobility drift region was described in experiment 3 in the previous section of this paper. Longer mobility drift paths could be implemented to aid in further discrimination between ions and neutrals than demonstrated here, operating with high electric fields and stacked ring electrodes similar to the mobility cell described by Gillig et al. [24]. The general source design concept in Figure 1 lends itself to these approaches where focusing elements can be implemented to filter contaminants in the high-pressure source region. With this concept, where the vacuum gate isolates the analyzer and the entire source region is opened for target access, routine replacement of these elements could be as readily achieved as replacing the MALDI target if plug-in voltage connections were utilized.

\section{Electrospray Versus MALDI}

As with all techniques, absolute sensitivity and $\mathrm{s} / \mathrm{n}$ measurements are highly dependant on the compounds measured, sample preparation, and method of acquisition. For the purposes of this discussion a comparison

\section{(a)}

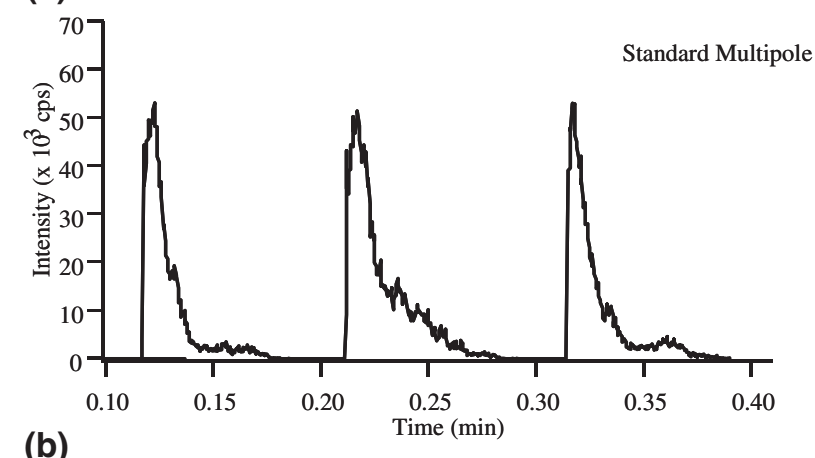

(b)



Figure 7. (a) Peak transients observed with a high-pressure RF multipole ion guide. (b) Peak transients observed with a directed gas flow along the length of the multipole ion guide. Laser pulse frequency was $1 \mathrm{KHz}$ in both cases. 




Figure 8. Image of a sample spot $50 \%$ ablated. The entire $0.5 \mathrm{uL}$ spot was consumed to obtain a measure of the ion yield for a sample of Reserpine monitoring the 609->195 transition.

of MALDI and electrospray efficiency of ion creation and sampling, as well as limits of detection is presented under the narrowly defined conditions used in this paper. The acquisition method used for MALDI is the method whereby the maximum acquisition speed is achieved by illuminating a single area of a sample spot. Electrospray data are presented operating in two different modes. The first, and of most general interest for this application, is the most widely accepted methodology for fast quantitation being LC/MS/MS operating at a flow rate of $200 \mu \mathrm{L} / \mathrm{min}$. The second is electrospray operating in the nanoflow regime where the ion transmission efficiencies are higher for this mode of ionization.

To obtain an efficiency measurement for MALDI, a spot of known quantity was entirely consumed by continuous rastering and re-rastering. Although this is not an analytically useful approach for fast quantitation, it is necessary for this particular measurement. Figure 8 is an image of a sample of 0.25 pg Reserpine $50 \%$ ablated to illustrate the experiment. The resulting ion current from product ion at $\mathrm{m} / \mathrm{z} 195$ was integrated yielding an average of 150,000 counts for five separate spots $(\sigma \pm 12 \%)$. The MALDI target was positioned 1.5 $\mathrm{mm}$ from the aperture to confine the ablation plume in the vacuum gas drag and transfer all ions into the ion guide. Although a precise ion current measurement has not been taken to confirm the totality of this transfer there are no obvious sources of loss. The loss of ions in MS/MS mode through this ion path has been well characterized for this compound [27]. Accounting for these losses and with a measurement of 150,000 ions arriving at the detector the number of ions entering $\mathrm{Q} 0$ can then be estimated at $\sim 1.2 \times 10^{7}$. Considering that $2.5 \times 10^{8}$ molecules of Reserpine were deposited on the target the ionization efficiency of MALDI observed here was $\sim 4.8 \%$ assuming $100 \%$ transfer efficiency into Q0 which is reasonable.
An equivalent amount of Reserpine was flow injected (5 replicates, $\sigma \pm 8 \%$ ) into a standard API 4000 instrument (identical ion path from Q0 on) equipped with an ion source adapted for good ionization efficiency at high electrospray flow rates (Turbo- $\mathrm{V}$ ion source). This system injects high-temperature nitrogen gas $\left(8 \mathrm{~L} / \mathrm{min}\right.$ at $\left.600{ }^{\circ} \mathrm{C}\right)$ into the entrainment region of a pneumatically assisted electrospray source to improve both ionization and transfer efficiency at high flow rates. The liquid flow rate was $200 \mu \mathrm{L} / \mathrm{min}$. The average integrated area counts were $6 \%$ of that achieved from the MALDI experiment or a factor of $\sim 15 \times$ fewer ion counts. Using the same efficiency calculations as above, the combined efficiency of ion creation and transfer through the atmospheric orifice was $0.31 \%$. Separating the ionization efficiency from the efficiency of transfer through the inlet atmospheric orifice is experimentally difficult and will be discussed with the nanospray results but suffice it to say that the majority of these losses are at the orifice. The electrospray ionization efficiency is high, even at these flow rates where many large droplets are formed and heating is used to rapidly reduce droplet diameters to ion emission dimensions.

When a fused silica nanospray emitter operating at a flow rate of $1000 \mathrm{~nL} / \mathrm{min}$ is inserted into the vacuum gas drag region equipped with an interface optimized for low flow rates [28], the area counts for an equivalent amount of sample are improved by a factor of $15 \times$ over the high flow electrospray experiment. This flow rate, although high for nanospray and not optimum for maximum transfer efficiency, was chosen because spraying this volume of liquid close to the vacuum orifice has no deleterious effect on the mass spectrometer and because of the practicality of this flow for chromatography purposes versus low $\mathrm{nL} / \mathrm{min}$ flows. The combined efficiency of ionization and orifice transfer is similar to the MALDI ionization efficiency, $4.8 \%$. Separating the ionization from orifice transfer efficiency can be estimated by measuring non-mass resolved ion current at the atmospheric side of the orifice and referencing it to the current arriving at a grounded element on the vacuum side, but these measurements can be ambiguous because they do not distinguish ions from charged particles and clusters. Experiments with a different vacuum arrangement allowing for an increase of the orifice area lead to a signal gain with this nanospray experiment. This leads to the conclusion that the majority of the ion losses in this relatively high flow nanospray experiment are occurring during transfer and not at the ionization step. Opening the aperture will let more ions through but will not create more ions. One can also conclude that the ionization efficiency of electrospray is considerably higher than MALDI but these gains are negated by limitations in transfer efficiencies from atmosphere to vacuum with this system and under these conditions. Values for electrospray ionization and transfer efficiency, operating at $100 \mathrm{~nL} / \mathrm{min}$ or less, have been measured to be as high as 50\% [29], 
albeit with considerable variability ranging from 0.5 to $50 \%$, the spread attributable to variations in sprayer geometry. There are thus sensitivity gains still available for nanoelectrospray above those described in this paper $(4.8 \%)$ provided they can be captured in an analytically useful and reproducible manner, preferably with chromatography in the low $\mu \mathrm{L} / \mathrm{min}$ flow range.

From an analytical standpoint there is more to consider than efficiency of ion creation and transfer. The size of the ablation area that can be ionized at one time has practical limitations in MALDI and for this instrument the illumination area has been optimized at 150 $\mu \mathrm{m}$ as described earlier. Given typical spot sizes of $2-2.5 \mathrm{~mm}$ from sample volumes of $1-2 \mu \mathrm{L}, \sim 0.5 \%$ of the sample can be ionized in the time frame of a rapid burn through of a single illumination area to obtain a quantitative measurement. Thus the 15-fold efficiency gain cited for MALDI over the high flow electrospray situation is negated by a 200-fold sampling deficit because of the low amount of sample available for ionization at one time. The MALDI absolute sensitivity and $\mathrm{s} / \mathrm{n}$ for this compound, under this acquisition scheme, was $\sim 15$-fold lower than the high flow electrospray experiment when one considers the total amount of sample spotted on the MALDI target versus the amount injected into an LC/ESI system at conventional flow rates.

Variations in the chemistries of individual compounds and chemical background noise at particular mass transitions will modify these estimates in particular cases but in general what has been observed over a large suite of drug like molecules is compounds that ionize efficiently with both electrospray and MALDI will demonstrate a 10- to 15-fold lower limit of quantitation with the conventional high flow LC/MS electrospray experiment. Methods to concentrate large-volume spots and/or increase ablation areas without loss in the optics will improve the situation for MALDI. However, the important MALDI advantage is speed of analysis. These observations are in alignment with the general findings of Gobey et al. [4] and Sleno and Volmer [5], whose tests were done over a much larger suite of compounds and conditions.

\section{$L C / M A L D I$}

The above analysis conjures up one obvious question. Can a larger percentage of the available sample be ionized by MALDI if the samples were concentrated into a smaller area? One analytically useful means of achieving this is through the use of efficient liquid chromatography combined with a means of depositing the eluant in a manner that does not lead to spreading on the target [30]. Experiments were performed here comparing LC/ESI with LC/MALDI to see if the combined concentration effect of chromatography and ablation of more sample by a single raster of the laser beam across the LC peak would improve the sensitivity and detection limits.
The $200 \mu \mathrm{L} / \mathrm{min}$ chromatographic conditions (2 mm i.d. column) used for the LC/ESI were chosen because they are commonly used for bioanalytical applications and the sensitivity differences obtained under these conditions versus $50 \mu \mathrm{L} / \mathrm{min}$ (those used for LC/ MALDI) are negligible with the Turbo-V source (operation conditions described earlier). To record the LC/ MALDI chromatograms a gas nebulizer was used to deposit the eluant on a target in a continuous trace at a flow rate of $50 \mu \mathrm{L} / \mathrm{min}$ using a column of $1 \mathrm{~mm}$ diameter. This chromatographic system was chosen to closely approximate the high flow electrospray conditions and offer the practical column loading and routine fluid connection advantages over a capillary LC approach. Also, spreading of the eluant on the target can be controlled with the nebulizer approach at these flows but becomes more problematic at higher flows. The stage was translated at a rate of $0.2 \mathrm{~mm} / \mathrm{s}$ resulting in a conversion of a $1 \mathrm{~min}$ chromatogram to a $12 \mathrm{~mm}$ trace. The laser was rastered continuously across the trace at a rate of $4 \mathrm{~mm} / \mathrm{s}$. Under these conditions the majority of a $6 \mathrm{~s}$ wide peak (measured at base) is deposited over a $0.8 \mathrm{~mm}$ diameter track, providing a theoretical maximum peak capacity of $15 / \mathrm{min}$. A single raster through this area consumes $\sim 10 \%$ of the sample, more than enough to compensate for the losses by the dried droplet method with illumination of a single laser area.

With this improvement in sample consumption, high flow LC/ESI and LC/MALDI should produce roughly equivalent sensitivities. This was observed to be the case as shown in Figure 9, where similar sensitivities and $\mathrm{s} / \mathrm{n}$ were obtained for a $50 \mathrm{fg}$ injection of Glyburide. Further reduction of the dispersion of the peak trace on the target occurs at lower flow rates such that at $5-10 \mu \mathrm{L} / \mathrm{min}(0.3 \mathrm{~mm}$ i.d. columns) the majority of the sample can be ablated in a single laser pass. In so doing LC/MALDI at these flows approaches the sensitivities observed from nanoLC electrospray operating at flows around $1 \mu \mathrm{L} / \mathrm{min}$, in keeping with the near equivalence of the ion production and transfer processes of these two techniques as described above. One advantage of LC/MALDI is that columns of relatively large diameters ( $0.3 \mathrm{~mm}$ diameter) and loading capacities can be used to increase the dynamic range and concentration sensitivity of an analysis whereas nanoLC/ESI achieves peak performance at flows less than $1 \mu \mathrm{L} / \mathrm{min}$ and smaller column diameters.

The data in Figure 9 also highlight the speed aspect of MALDI emphasized in this paper. The 1 min chromatographic time in Figure 9a was read by the laser in $3 \mathrm{~s}$. In other words, the mass spectrometry acquisition time is 20 times faster than the chromatography. In this case the total sample throughput does not change, as it is limited by the chromatography; however this presents a reasonable case to parallelize chromatographic systems for MALDI target preparation where maximum advantage would be achieved with 20 chromatograms prepared simultaneously. 

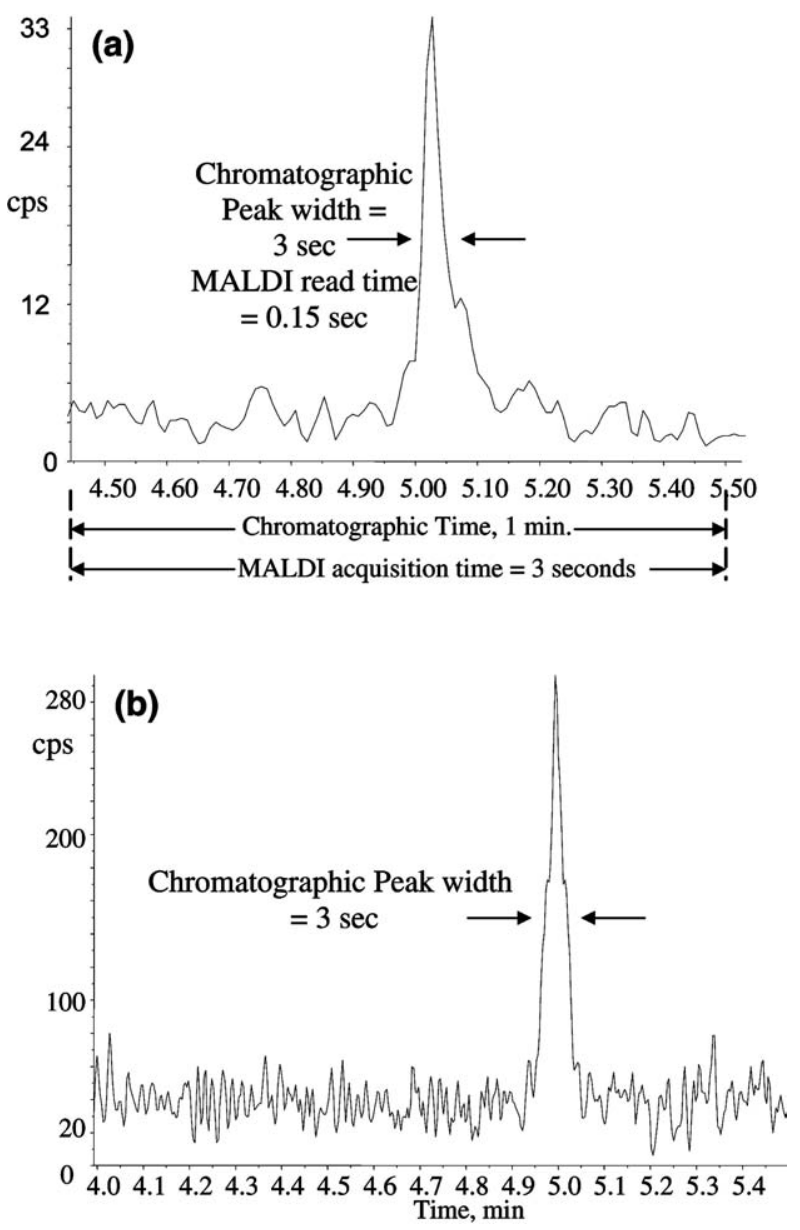

Figure 9. Comparison of LC/MALDI with high flow LC/ESI on a $50 \mathrm{fg}$ injection of Glyburide monitoring the MRM transition $369 \rightarrow 169$ at a laser pulse frequency of $1400 \mathrm{~Hz}$ and energy of 4.5 $\mu \mathrm{J} /$ pulse. (a) LC/MALDI data obtained from track deposited at 50 $\mu \mathrm{L} / \mathrm{min}$ and $1 \mathrm{~mm}$ diameter column. (b) LC/ESI at $200 \mu \mathrm{L} / \mathrm{min}$ with a $2 \mathrm{~mm}$ diameter column.

\section{Summary and Conclusions}

An instrument is described with the theoretical potential to achieve quantitative measurements about 10 samples per second, this limitation being incurred by spreading of the ion beam in the ion path. Limits imposed by sample preparation, sample loading, and stage movement are not considered in this calculation. The merits of a triple quadruple analyzer for this purpose are described and the requirements of a MALDI ion source and mass spectrometer interface specific for this application, significantly distinct from traditional MALDI applications and source requirements, are presented in detail. The relative sensitivities of MALDI and electrospray for this quantitation application are compared on a common analyzer system.

In this regard, the primary findings can be summarized as follows:

1. Collisional cooling with low torr pressures is essential to reduce fragmentation of fragile small molecules and increase the useful fluence range.
2. A laser with low power $(<10 \mu \mathrm{J} /$ pulse $)$ and high pulse frequency $(\mathrm{kHz})$ is critical to achieve high sampling speed and high signal to noise ratios.

3. Ion beam spreading defines the theoretical maximum throughput attainable, which is 10 samples per second limit with this instrument.

4. Sample-to-sample carry-over effects exist with MALDI and are addressed with laser angle of incidence.

5. Transporting ions with gas flows and electric fields are optimized to discriminate between ions and contaminating neutrals.

6. Operating MALDI in the high throughput mode described here results in limits of detection 10-15× worse than conventional LC/ESI due to the low sampling efficiency of MALDI. The MALDI advantage is speed of acquisition. Methods to concentrate sample spots on targets improve the detection. One means to achieve this is with LC peak concentration on MALDI targets and, under the conditions described here, approximately equivalent detection to conventional LC/ESI techniques is observed.

\section{References}

1. Krutchinsky, A. N.; Loboda, A. V.; Spicer, V. L.; Dworschak, R.; Ens, W.; Standing, K. G. Orthogonal Injection of Matrix-Assisted Laser Desorption/Ionization Ions into a Time-of-Flight Spectrometer Through a Collisional Damping Interface. Rapid Commun. Mass Spectrom 1998, 12, 508-518.

2. Bucknall, M.; Fung, K. Y. C.; Ducan, M. W. Practical Quantitative Biomedical Applications of MALDI-TOF Mass Spectrometry. J. Am. Soc. Mass Spectrom 2002, 13, 1015-1027.

3. Cohen, L. H.; Gusev, A. I. Small Molecule Analysis by MALDI Mass Spectrometry Anal. Bioanal. Chem. 2002, 373, 571-586.

4. Gobey, J.; Cole, M.; Janiszeski, J.; Covey, T.; Chau, T.; Kovarik, P.; Corr, J. Characterization and Performance of MALDI on a Triple Quadrupole Mass Spectrometer for Analysis and Quantification of Small Molecules. Anal. Chem. 2005, 77, 5643-5654.

5. Sleno, L.; Volmer, D. A. Some Fundamental and Technical Aspects of the Quantitative Analysis of Pharmaceutical Drugs by Matrix-Assisted Laser Desorption/Ionization Mass Spectrometry. Rapid Commun. Mass Spec. 2006, 19(14), 1928-1936.

6. Chernushevich, I. V. Duty Cycle Improvement for a Quadrupole-Timeof-Flight Mass Spectrometer and Its Use for Precursor Ion Scans. Eur. J. Mass Spectrom. 2000, 6, 471-479

7. Hager, J. W. A New Linear Ion Trap. Rapid Commun. Mass Spectrom. 2002, 16, 512-526.

8. Thomson, B. A.; Jolliffe, C.; Javahery, R. RF-Only Quadrupoles with Axial Fields. Proceedings of the 44th ASMS Conference on Mass Spectrometry and Allied Topics; Portland, Oregon, May, 1996.

9. Lock, C. M.; Dyer, E. Characterization of High Pressure Quadrupole Collision Cells Possessing Direct Current Axial Fields. Rapid Commun. Mass Spectrom. 1999, 13, 432-448.

10. Schneider, B. B.; Lock, C. M.; Covey, T. R. AP and Vacuum MALDI on a QqLIT Instrument. J. Am. Soc. Mass Spectrom. 2005, 16, 176-182.

11. Douglas, D. J.; French, J. B. Collisional Focusing Effects in Radio Frequency Quadrupoles. J. Am. Soc. Mass Spectrom. 1992, 3, 398-408.

12. Ericson, C.; Phung, Q. T.; Horn, D. M.; Peters, E. C.; Fitchett, J. R.; Ficarro, S. B.; Salomon, A. R.; Brill, L. M.; Brock, A. An Automated Noncontact Deposition Interface for Liquid Chromatography MatrixAssisted Laser Desorption/Ionization Mass Spectrometry. Anal. Chem. 2003, 75, 2309-2315.

13. Paul, P.; Rehm, J.; Covey, T.; Kovarik, P.; Yang, M.; Flarakos, T. High Resolution LC/MALDI System. Proceedings of the 53rd ASMS Conference on Mass Spectrometry and Allied Topics; San Antonio, Texas, May, 2005.

14. Hanton, S. D.; Liu, X. M. GPC. Separation of Polymer Samples for MALDI Analysis. Anal. Chem. 2000, 72, 4550-4554.

15. Verentchikov, A. N., Vestal, M. L., Smirov, I. P. Method and Apparatus for Determining Molecular Weight of Labile Molecules. U.S. Patent No. 6,504,150 B1, January 2003

16. Verentchikov, A.; Smirnov, I.; Vestal, M. Collisional Cooling and Ion Formation Processes in Orthogonal MALDI at Intermediate Gas Pressure. Proceedings of the ASMS Conference on Mass Spectrometry and Allied Topics; Dallas, Texas, June, 1999.

17. Loboda, A. V.; Ackloo, S.; Chernushevich, I. V. A High-Performance Matrix-Assisted Laser Desorption/Ionization Orthogonal Time-of- 
Flight Mass Spectrometer with Collisional Cooling. Rapid Commun. Mass Spectrom. 2003, 17, 2508-2516.

18. Loboda, A. V.; Chernushevich, I. V. Investigation of the Mechanism of Matrix Adduct Formation in MALDI at Elevated Pressure. Int. J. Mass Spectrom. 2005, 240, 101-105.

19. McLean, J. A.; Russell, W. K.; Russell, D. H. A High Repetition Rate (1 $\mathrm{kHz}$ ) Microcrystal Laser for High Throughput Atmospheric Pressure MALDI-Quadrupole-Time-of-Flight Mass Spectrometry. Anal. Chem. 2003, 75, 648-654.

20. Westman, A.; Huth-Fehre, T.; Demirev, P.; Bielawski, J.; Medina, N.; Sundqvist, U. R. Matrix-Assisted Laser Desorption/Ionization: Dependence of the Ion Yield on the Laser Beam Incidence Angle. Rapid Commun. Mass Spectrom. 1994, 8, 388-393.

21. Aksouth, F.; Chaurand, P.; Deprun, C.; Della-Negra, S.; Hoyes, J.; Le Beyec, Y.; Rosas Pinho, R. Influence of the Laser Beam Direction on the Molecular Ion Ejection Angle in Matrix-Assisted Laser Desorption/ Ionization. Rapid Commun. Mass Spectrom. 1995, 9, 515-518.

22. Puretzky, A. A.; Geohegan, D. B.; Hurst, G. B.; Buchanan, M. V.; Luk'yanchuk, B. S. Imaging of Vapor Plumes Produced by Matrix Assisted Laser Desorption: A Plume Sharpening Effect. Phys. Rev. Lett. 1999, 82(2), 444-447.

23. Ashkeas, H.; Sherman, F. S. The Structure and Utilization of Supersonic Free Jets in Low Density Wind Tunnels. In Fourth International Symposium on Rarefied Gas Dynamics; Deleeuw, J. H., Ed; Academic Press: New York, 1992, pp 613-650.
24. Gillig, K. J.; Ruotolo, B.; Stone, E. G.; Russell, D. H.; Fuhrer, K.; Gonin, M.; Schultz, A. J. Coupling High-Pressure MALDI with Ion Mobility/ Orthogonal Time-of-Flight Mass Spectrometry. Anal. Chem. 2000, 72, 3965-3971.

25. Loboda, A.; Krutchinsky, A.; Loboda, O.; McNabb, J.; Spicer, V.; Ens, W.; Standing, K. G. Novel LINAC II Electrode Geometry for Creating an Axial Field in a Multipole Ion Guide. Eur. J. Mass Spectrom. 2000, 6(6), 531-536.

26. Dodonov, A.; Kozlovsky, V.; Loboda, A. Raznikov, V. Sulimenkov, I.; Tolmachev, A.; Kraft, A.; Wollnik, H. A New Technique for Decomposition of Selected Ions in Molecule Ion Reactor Coupled with OrthoTime-of-Flight Mass Spectrometry. Rapid Commun. Mass Spectrom 1997, $11,1649-1656$.

27. Thomson, B. A.; Douglas, D. J.; Corr, J. J.; Hager, J. W.; Jolliffe, C. L. Improved Collisionally Activated Dissociation Efficiency and Mass Resolution on a Triple Quadrupole Mass Spectrometer System. Anal. Chem. 1995, 67(10), 1696-1704.

28. Schneider, B. B.; Baranov, V. I.; Javahari, H.; Covey, T. R. Particle Discriminator Interface for Nanoflow ESI-MS. J. Am. Soc. Mass Spectrom. 2003, 14, 1236-1246.

29. El-Faramawy, A; Siu, M.; Thomson, B. A. Efficiency of Nano-Electrospray Ionization. J. Am. Soc. Mass Spectrom. 2005, 16, 1702-1707.

30. Zhang, B.; McDonald, C.; Li, L. Combining Liquid Chromatography with MALDI Mass Spectrometry Using a Heated Droplet Interface. Anal. Chem. 2004, 76, 992-1001. 